\title{
Review of: "Evidence for increased breakthrough rates of SARS-CoV-2 variants of concern in BNT162b2 mRNA vaccinated individuals"
}

\author{
Peter Mclntyre ${ }^{1}$ \\ 1 University of Otago
}

Potential competing interests: The author(s) declared that no potential competing interests exist.

This is an interesting and important study of breakthrough infections with SARS-CoV-2 in individuals who have had either one dose or two doses of BNT162b2 mRNA vaccine and attended a large health management organisation (Clalit Health Services) in Israel which has published several post-marketing studies of BNT162b2 from amongst its attendee population, which includes over 4 million members. In Israel during the study period the UK-derived B1.1.7 strain was by far predominant, accounting for more than $90 \%$ of PCR-positive viral identifications genotyped. This significantly limited the power of the study to examine differences in relative proportions of the B1.351 and wild type virus versus B1.1.7 as the former had almost disappeared by half way through the study period in the general population. Study methodology was high quality, both for procedures for selection of unvaccinated controls by date of onset and for genotyping methods, although the authors acknowledge that age-matching was least satisfactory given that most vaccinated individuals were in older age groups and most unvaccinated individuals in younger age groups. This relatively poor age-matching can be clearly seen in the study Table and indeed the analysis for relative risk of B1.351 infection rests on only 8 discordant pairs. Although there were substantial numbers of two-dose vaccinated cases (149), only 46 of them occurred at least 21 days after receipt of the second dose. We are not given any information about the clinical presentation or symptom severity of either two or one dose recipients. This is crucial, because it is not surprising that there would be PCR positive infections demonstrated in fully vaccinated individuals in a large population where the virus is still circulating, if these people all have mild or no symptoms who are being identified because of high rates of testing of any individual with respiratory symptoms, this is of far less concern than more severe breakthrough infections, whether they be due to VOCs or wild strain virus. The main value of this study in my view is proof of principle and as a template for investigation of breakthrough cases and identification of relative genotype distribution amongst them. Although the authors demonstrated a statistically significantly higher proportion of B1.135 amongst two dose recipients, the vast majority of them were infected by the dominant B1.1.7 strain. That the small proportion of B1.135 circulating strains are more likely to infect two dose recipients than unvaccinated persons, especially if the latter are predominantly among younger adults with very different mixing patterns, is unsurprising. The data presented here do not suggest that this phenomenon is of any public health significance in Israel but do provide a template for appropriately resourced settings, especially where a number of different COVID-19 vaccines are being used, to investigate breakthrough infections 
